\title{
Research on Learning Ability Evaluation Model of Educational Virtual Community Members
}

\author{
Yan Cheng, Zhiming Yang, and Yan Zeng
}

\begin{abstract}
Owing to the advanced information technologies, the development of knowledge update very quickly .Especially in educational environment of e-learning, the learning ability is a very important indicator for the learners. Educational virtual community provides an open platform of interactive learning for community members. In this paper, the authors discussed the existing methods that the evaluation of learning ability, and constructed the evaluation model of students' learning ability to quantitative evaluation of the ability. The details of learning ability are classified into independent learning ability and interactive learning ability. Based on the fuzzy comprehensive evaluation, a learning ability evaluation model is proposed to realize more accurate assessment of the learner's ability, and the result of the research will be able to apply to computer assisted education to support for the teaching management.
\end{abstract}

Index Terms - Educational virtual community, independent learning, interactive learning ability, fuzzy comprehensive evaluation.

\section{INTRODUCTION}

With the demanding of "just-in-time training" for globally employees, the traditional classroom training can't meet the requirement of people. Most of them have switched to virtual e-learning and other ubiquitous ways of learning [1]. In modern society, the key to win is the ability to compete in the era of knowledge economy. Learning ability is one of the important marks for the 21 st century talents. The traditional study of human knowledge score is not enough for the present study mode. Learning ability evaluation of students is an important means of teaching management to the related teaching department. It gets very necessary and urgent to evaluate students learning abilities.

In recent years, the existence and development of online virtual communities affect the real life of college students.

The educational virtual community members will conduct special training, mutual cooperation and resource sharing.

In this paper, the authors summarized the existing methods that the evaluation of learning ability, and constructed the evaluation model of students' learning ability. For the

Manuscript received September 6, 2015; revised October 23, 2015. This work was supported by National Natural Science Foundation of China (NSFC, Grant No. 61262080), Jiangxi Province Science and Technology Support Major Project (20151BBE50121), and 2011 collaborative innovation center "Based on Big Data of Jiangxi Province Teacher Quality Monitoring, Evaluation and Service".

Yan Cheng is with Tongji University. She is also with Jiangxi Normal University, Nanchang, Jiangxi, China (e-mail: chyan88888@jxnu.edu.cn).

Zhi Ming Yang and Yan Zeng are with Jiangxi Normal University, Nanchang, Jiangxi, China (e-mail: 709830862@qq.com, ningmoxi@163.com). problem of fuzzy degree of evaluation results, which based on the fuzzy comprehensive evaluation, we put forward a learning ability evaluation model to realize more accurate assessment of the learner's ability.

\section{ABILITY EVALUATION METHOD}

For a long time, people often used description language to evaluating ability, such as "almost", "generalization" and so on. The result is lack of scientific nature, which will directly affect the evaluating effectiveness and also hinder the cultivation of the talent [2]. The process of learning ability evaluation is also inevitably related to the subjective judgment which often relies on teachers' subjective judgment.

According to the concept that fuzzy imprecise information to processing the human subjective quantitative, the fuzzy theory can be used to represent the level of teachers' grading [3]. Comprehensively considers the contributions of multiple related indicators according to weights and decreases the fuzziness by using membership functions [4].

In order to solve these uncertainties, fuzzy comprehensive evaluation is used to evaluate an objective by the fuzzy set theory. Reference [5] presents an interval evidential reasoning (IER) approach for autonomous learning ability assessment, which uses the analytical evidential reasoning (ER) algorithm to aggregate all evidence simultaneously. But the learning evaluation, did not fully reflect the E-learning interactive collaboration, only consider E-learning autonomy. With the rapid development of the information society, reference [6] puts forward new requirements for the professional talent training in economic management profession in colleges. This thesis discussed the evaluation index system of student's ability of using information technology in economic management profession, and given a fuzzy synthesis evaluation model. Because of many related factors in occupational ability, researchers in China lack the corresponding exploration and research on factors organic integration and working out a convincing evaluation result.

The article [7] combined fuzzy evaluation method with level evaluation method and applied them to evaluating occupational ability so as to provide some beneficial explorations for it. Results obtained by different fuzzy evaluation model evaluation ambiguity have some varies. That the method to make fuzzy evaluation model evaluation results for a smaller ambiguity is worth studying. The evaluation of scientific and technological talents is a very important job, the paper [8] have given the solution of two specific aspects aiming at the practice of talents evaluation. They used optimization method to solve performance ability in evaluation about the organic combination of problems. The 
Bayesian method is used to solve the evaluation of the effect of tracking and correction problem. The rating of learners' comprehensive ability of community should neither simply assessed by test scores, nor simple comment to the students. Such an evaluation, which most of the network prior learning assessment, is limited to the online test scores to evaluate .In order to solve the fuzzy evaluation model evaluation results which lead to greater ambiguity and maximum subordination principle that may lead to distortion of the evaluation results and other issues, this paper brings full advantage of comprehensive evaluation of the information presented and establish level membership quantitative model to further quantify the results of the evaluation.

The key steps of Fuzzy comprehensive evaluation method as follow:

1) Determine factor set and evaluating set.
2) Give the distribution of the weight of each factor.

3) Determine the multi-factor fuzzy evaluation matrix R.

4) Calculate evaluation result set.

5) Determine the fuzzy comprehensive evaluation results.

\section{The PRocess OF LEARNING ABILITy EVAluAtion}

Based on the characteristics of network teaching, evaluating comprehensively mainly from the following three aspects, namely learning enthusiasm, expertise level and competence level of community learners by combing practical experience in teaching. Through these evaluations, the learning performance assessment indicator system of online community was established. As shown in Fig. 1:

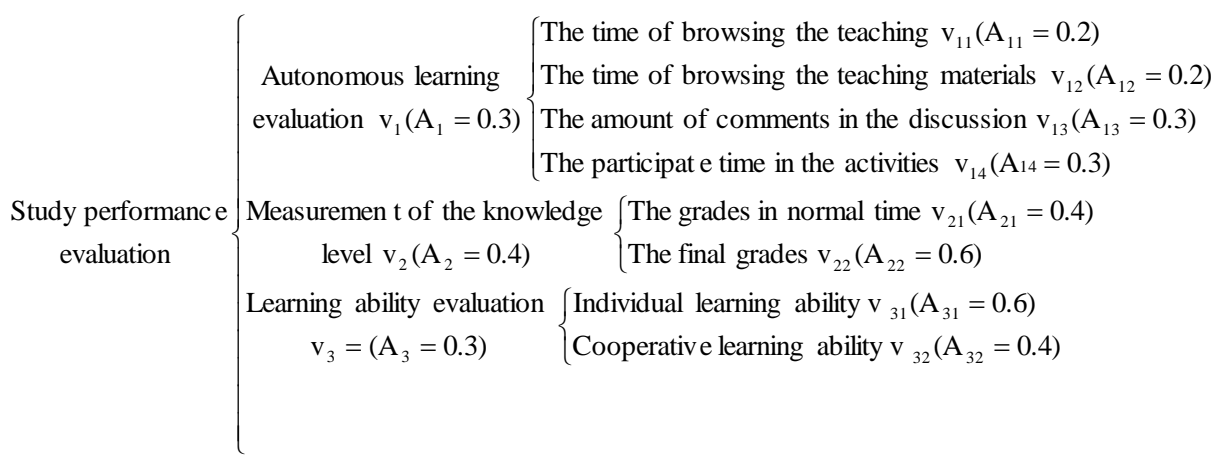

Fig. 1. The structure of learning community performance evaluation system.

Evaluation factors set is defined as $V=\left\{v_{1}, v_{2}, v_{3}\right\}$, Among which $v_{1}=\left\{v_{11}, v_{12}, v_{13}, v_{14}\right\}, v_{2}=\left\{v_{21}, v_{22}\right\}$, $v_{3}=\left\{v_{31}, v_{32}\right\} \cdot v_{i}$ represents for the evaluation factors, and $A_{i}$ is for the corresponding weights; $u_{i j}$ represents for its lower level evaluation factor, and $A_{i j}$ is for the corresponding weights. Limited by the length, the process of how to weigh and determine the weights of evaluation factors won't be described here. Assume that the values of the factors weights $A_{i}, A_{i j}$ as shown in Fig. 1.

\section{A. The Assessment of Community Learning Knowledge Level}

Through the test the knowledge level of learners can preliminary be grasped. But in some condition, in order to be more accurately distinguish students, the additional information to achieve a goal is need to be added. In actual network teaching, the students' grades will be recorded, such as homework or tests at ordinary times. The grades also reflects the student's scores. So taking the grades into consideration is also reasonable. At the same time, the final grade can be modified by actual grades that can more objectively to reflect the students' knowledge level. By adopting fuzzy set theory, this fuzzy information is still need to be handled.

Definition 1: $L=\left\{l_{1}, l_{2}, l_{3}, \cdots, l_{n}\right\}$ represents the collection of course; $l_{i}$ represents a student's courses in a term; $R=\left\{r_{1}, r_{2}, r_{3}, \cdots, r_{n}\right\}$ represents the collection of level ; $r_{j}$ represents the number of the level $, j=1,2,3, \cdots, m$, $T=\left\{t_{1}, t_{2}\right\}$ is the collection of test, $t_{1}$ represents the result of the actual test or homework ; $t_{2}$ represents the test of the final term .

According to the fuzzy set theory, a weight vector is needed to illustrate each of the students belonging to which level. And then classify the students to one of the level using the fuzzy theory .The algorithm steps are as follows: 1) According to student's usual grades level to establish the grades of initial matrix $R^{(1)}=\left(r_{i j}^{(1)}\right)$. Among which $r_{i j}^{(1)}=n_{j} /$ total represents the number of test of the student's usual grades which is $r_{j}$ in one of the courses; total represents the sum of the usual test. 2) Based on the student's final grade, the final result of initial matrix $R^{(2)}=\left(r^{(2)}{ }_{i j}\right)$ can be made,

$$
r_{i j}^{(2)}=\left\{\begin{array}{l}
1, \text { if the level of course } l_{i} \text { is } r_{j} \\
0, \text { if the level of course } l_{i} \text { isn't } r_{j}
\end{array}\right.
$$

To determine the test weight vector $W_{t}=\left(w_{t 1}, w_{t 2}\right)$ and weight vector of the curriculum $W_{l}=\left(w_{l 1}, w_{l 2}, \cdots, w_{l n}\right)$. According to the experience, the vector $W_{t}=(0.6,0.4)$ can be made to the actual situation and also combine students' professional situation. 4) If $r_{i j}=\sum_{k=1}^{2} w_{t k} * r_{i j}^{(k)}, R=\left(r_{i j}\right)$ is judgment matrix. 5) If $S_{u_{2}}=\left(s_{1}, s_{2}, s_{3}, \cdots, s_{m}\right)$ represents the fuzzy 
comprehensive evaluation vector, $S_{u_{2}}=W_{l} \cdot R$ If $S_{k}$ is the maximum number of the assemble $\left\{S_{k}\right\}$, the students belong to $r_{k}$; According to the theory of fuzzy maximum membership principle, if there are several equals to the maximum, we may choose the highest level as approximation.

\section{B. Independent Learning Ability Evaluating}

Mastering the knowledge is a gradual process. In general, students' independent learning ability is mainly reflected in the process to solve the problem, and students' independent learning ability is dynamic change. According to the principle of maximum membership, in the final membership matrix, the comprehensive index of which the degree of membership is higher, then we will be the target set for evaluation. Fuzzy set way is used to represent students' independent learning ability, and the maximum membership degree method to determine the level of knowledge levels. For example: 5 level membership of a student ability level is: $\{0.2,0.3,0.1,0.2$, $0.2\}$, level 2 is the highest degree of membership which can be drawn from the students' ability to achieve the third level.

To evaluate the independent of the students' ability of learning, we will consider the student's mastery of knowledge and two factors: do question time and difficulty of the questions. Question time used to measure students' mastery. Provides two time periods: normal and maximum time. Students do questions right at the usual time, that mastery of knowledge to a higher level; between normal and maximum time to make a question, you can grasp the general level of knowledge; if over the maximum time for knowledge mastery lesser extent. That the students' mastery of knowledge itself is ambiguous, so the membership functions is used to represent the learners' knowledge levels. In this paper, mastery of knowledge of the membership function $K(t)$ is defined as follows :

Definition 2: $F(t)$ is a membership function which represents mastery degree of the knowledge of the student: $t$ represents the answer time, $\mathrm{n}$ represents the normal time, 1 represents the longest time,

$$
F(t)=\left\{\begin{array}{l}
1 \quad t \leq n \\
\frac{l-t}{l-n} \quad n<t \leq l \\
0 \quad t>l
\end{array}\right.
$$

Along with the increase of the value of the function $F, t$ is monotone decreasing, namely the students' mastery degree is diminishing by time. When students answer the questions in the normal period of time, $F(t)=1$; When the student answer the questions out of the longest time, $F(t)=0$.

According to the situation of answering the questions, to present the learning ability, fuzzy set membership changes using the thought of Sherlock II [9]. Sherlock II is a system that a fuzzy set that can achieve user status change and uses fuzzy sets to an awareness of the problem which is divided into several levels. Through each level to determine the degree of membership of a different understanding of the situation, considering the difficulty level and the time to do problems, forming the changing rules of Learning membership:

\section{1) Answer is right}

Students do problem right, the master of the problem would increases, the corresponding increase in high-level membership, low levels of membership decreased. different levels of questions have different effects to the student on mastering knowledge.

Definition 3: the learning ability of students is classified as low, little low, medium, high, very high level of five (ASC), respectively, with 1 to 5 to represent. Each level of membership is expressed as $\mu_{S}(i), i=1 \sim 5$, student learning fuzzy sets represented by $S$. D represents it which is divided into five levels: difficult, more difficult, medium and relatively easy, easy, respected by : 1, 2, 3, 4, 5.The more difficult the value is the less difficulty they are. If the students make a difficulty for the level D questions, each level membership changes as follows:

$$
\left\{\begin{array}{c}
\mu_{S}(1)=\mu_{S}(1)-\mu_{S}(1) C \\
\mu_{S}(i)=\mu_{S}(i)-\left[\mu_{S}(i)-\mu_{S}(i-1)\right] C ; i=2,3,4 \\
\mu_{S}(5)=\mu_{S}(5)+\mu_{S}(5) C
\end{array}\right.
$$

Among them, $C=\lambda^{D} \cdot F(t), C$ is a content, $0<\lambda<1$, $D \in\{1,2,3,4,5\}$ (D represents the difficulty). When an right answer is to be done for the questions, the low level membership must fall, the high level membership will rise , intermediate level membership degree may rise or fall according to the value between $\mu_{S}(i)$ and $\mu_{S}(i-1)$. If $\mu_{S}(i)>\mu_{S}(i-1)$, the intermediate level membership degree may fall and vice versa. 1) From $C=\lambda^{D} \cdot F(t)$, the value of $C=\lambda^{D}$ is the biggest when the students finish the question in the normal time. The value of $\mathrm{C}$ will decrease with the decrease of the value of $F(t)$ when the students finish the question beyond the normal time; when the students finish the question beyond the longest time $C=0(F(t))$, the rising rules will not work. This reflects that the longer the student takes, the smaller the corresponding learning ability is. 2) when $F(x)$ is confirmed ,because $0<\lambda<1$, so the value of $\mathrm{D}$ is smaller (which means it's harder ); the bigger the value of $\lambda^{D}$ is , the bigger the value of $\mathrm{C}$ is . Namely the more difficult, the more high grade membership degree rise and reflects the difficulty for the faster, the greater the topic learning ability to rise.

\section{2) Answer is wrong}

Students do one question wrong, only consider its difficulty level (do question time is not important at this time), and the corresponding high-level membership decrease (if itself is 0 , still 0), low-level membership increases.

Definition 4: If the students do a question wrong which difficulty is D, each level of membership changes are as follows:

$$
\left\{\begin{array}{c}
\mu_{S}(1)=\mu_{S}(1)+\mu_{S}(1) C \\
\mu_{S}(i)=\mu_{S}(i)-\left[\mu_{S}(i)-\mu_{S}(i+1)\right] C ; i=2,3,4 \\
\mu_{S}(5)=\mu_{S}(5)-\mu_{S}(5) C
\end{array}\right.
$$


In the above formula, $C=\eta \cdot D, C$ is a constant , $0<\eta<1 / 5, H \in\{1,2,3,4,5\}$.

When students do a wrong topic, simply considering of the level D (the time is not important to the problem), and a corresponding high level of membership degree reduce ( if itself is 0 ,it is still 0 ), the low level of membership degree increase .The increasing or decreasing of the intermediate level membership depend on the value of $\mu_{S}(i)$ and $\mu_{S}(i+1)$. If $\mu_{S}(i)>\mu_{S}(i+1)$, the intermediate level membership is decrease and vise versa. The smaller $\mathrm{D}$ is (which represents it is easier), the greater the value of $\mathrm{C}$ is, the greater the difficulty of small problems to do the easier topic wrong, much lower level membership degree rise. It reflects the knowledge ability level falling fast for doing the easier problems wrong than doing difficult problems wrong.

The experimental results show that with the increase of doing problems, the level of learning ability which is obtained by this method was close to the students' actual level. At the same time, along with the increase of the practice, learners' learning ability level increases.

$=$ After the grade membership vector $\mathrm{S}$ is determined, the maximum membership degree method is used to determine the learning ability levels. But in many cases it need to be more accurately to distinguish students, such as finding a student's study ability of 5 level membership, like: $S_{u_{31}}=\mathrm{S}=$ $\{0.1,0.2,0.4,0.1,0.2\}$, and finding another student's learning ability of 5 level membership, like: $S_{u_{31}}=\mathrm{S}=\{0.1,0.1,0.1$, $0.1,0.2\}$, from which it can be concluded that the two students' learning ability level have reach the grade three . How to distinguish between students' learning ability further is also our issues to be solved. The level of quantitative models is adopted to solve this problem, which is in section 6 .

\section{Interactive Learning Ability Assessment}

According to the case the degree of interaction and collaboration in E-learning process, by the evaluations of learning communities or the other members of the team, in to determine the ability of the members of the collaborative learning, collaborative learning is according the evaluation of the members of the learning communities in communities' learning to determine. Collaborative learning ability of students evaluation is mainly reflected in four aspects: performance of teamwork and attitude, organizational skills, communication skills, teamwork responsibility; evaluation grade five, from low to high deepened, with 1 to 5 to represent Evaluation by a study group after a collaborative learning in collaborative learning partner for each factor separately scoring. Draw current collaboration evaluation matrix.

$$
R=\left[\begin{array}{lllll}
c_{11} & c_{12} & c_{13} & c_{14} & c_{15} \\
c_{21} & c_{22} & c_{23} & c_{24} & c_{25} \\
c_{31} & c_{32} & c_{33} & c_{34} & c_{35} \\
c_{41} & c_{42} & c_{43} & c_{44} & c_{45}
\end{array}\right]
$$

Among (5), $c_{i j} \in\{0,1\}, I=\{1,2,3,4\}$ ( $i$ denotes the evaluation factors),$j=\{1,2,3,4,5\}$ (j denotes the level of evaluation sets), $c_{i j}$ is a factor in favor of the first $I, j$ is the number of classes.

For each evaluation factor has a corresponding weight value (given by the teacher), set $w_{j}\left(0 \leq w_{j} \leq 1\right)$, The corresponding weight vector is: $w=\left(w_{1}, w_{2}, w_{3}, w_{4}\right)$, Thereby obtaining the cooperation on the student's ability to evaluate vector $S=Q=W \cdot R=\left(q_{1}, q_{2}, q_{3}, q_{4}, q_{5}\right)$.

\section{QuANTITATIVE MODEL FOR GRADE}

\section{A. Elementary Quantitative Model for Grade}

In the handling of some practical problems, in order to make full use of the comprehensive evaluation of information, visual evaluation results of the vector for a weight (normalized), will be judged on each level by 1 point or 100 points that make quantification, and then a weighted average of the evaluation results and total score can be obtained. Network learning ability and performance evaluation of fuzzy evaluation results for membership vector which can be further adopted to calculate the score. But if the set of numerical subjectively is valued by a subjective experience which will affect the accuracy of the results in a certain extent. That we should know how to reasonable quantitative evaluation set is also another important research.

\section{B. Accurate Quantitative Model for Level}

The quantitative common method used in front is to allocate each level, according to the experience or probably, a certain score. Five levels of evaluation sets: $\mathrm{V}=\{\mathrm{v} 1$ (good),v2(well), v3(general),v4(slightly poor), $\mathrm{v} 5$ (poor) $\}$, with a 1-100 points number is expressed as : $V=\{100,80,70,60,50\}$. Clearly, this quantitative method is not accurate enough ideal and also can't meet the actual needs. Therefore, how to reasonably quantify accurately distinguish between membership grade level of students is an important step. We get a suitable formula according to the fuzzy set theory, law of large numbers, and psychology. Defined as follows:

Definition 5: Suppose the feeling that a person is to an objective reality or to the change of a system is proportional to the objective things or system changes. If $S$ represents the feeling, T represents the objective things or system status. The rate of change of the objective state is $\Delta T / T$. According to the above hypothesis, we obtain as follows: $\Delta S=K \cdot \Delta T / T$. Integral equation is:

$$
S=K \cdot \ln T
$$

Namely the human sense and $T$ ratio pairs. Processed by the human brain, the qualitative levels are: $\mathrm{A}+, \mathrm{A}, \mathrm{A}-, \mathrm{B}+$, $\mathrm{B}, \mathrm{B}-, \ldots$ If $I$ represents the objective change in the form described, assuming: $T=f(I)=X \pm I$. When $\mathrm{X}$ is an uncertain variable, according to (6) we obtain:

$$
S(I)=K \cdot \ln f(I)=K \cdot \ln (X \pm I)
$$

Assume: $S \in[0,1], I=1,2,3, \cdots, j$. Use two boundary 
conditions:

$$
\begin{gathered}
I=1, S=1 \Rightarrow K \cdot \ln (X \pm 1)=1 \\
I=j+1, S=0 \Rightarrow K \cdot \ln [X \pm(j+1)]=0
\end{gathered}
$$

Solving equations (8), (9), get:

$$
\begin{gathered}
X=j+2 \\
K=1 / \ln (X-1)=1 / \ln [(j+2)-1]
\end{gathered}
$$

When the operator in the equation is + , the solution is not the real meaning. Assuming $m=j+2$, putting (10) into type (8), we can obtain the following functions:

$$
S(I)=\ln (X-I) / \ln (X-1)=\ln (m-I) / \ln (m-1)
$$

In the function above, $I=1,2,3, \ldots, j, S(I)$ and $\mathrm{I}$ is the value of the corresponding function, $j$ represents the number of grades, $\mathrm{m}$ is a general parameter. The results of this approach are believed to be reasonable. The function (11) represents the relationship between the quantitative value $S(I)$ and the qualitative value I . The number of levels $j=8,5$, 4, quantitative and qualitative value relationship $(S(1)=100 \%)$ as the following:

TABLE I: THE NUMBER OF LEVELS $\mathrm{J}=8$

\begin{tabular}{lllllllll}
\hline $\mathrm{I}$ & 1 & 2 & 3 & 4 & 5 & 6 & 7 & 8 \\
\hline $\mathrm{S}(\mathrm{I})(\%)$ & 100 & 95 & 89 & 82 & 73 & 63 & 50 & 31 \\
\hline
\end{tabular}

TABLE II: THE NUMBER OF LEVELS J=5

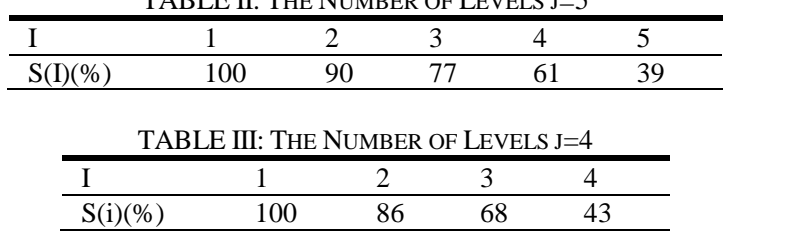

In some cases, it is not appropriate to value $S(1)$ as $100 \%$. Assuming that max is the highest $S(i)$, we can use the following formula to substitute (8) Formula:

$$
I=1, S=\max : K \cdot \ln (X \pm 1)=\max
$$

So this function is to be got:

$$
S(i)=(\max \cdot \ln (x-i)) / \ln (x-\max )
$$

For example: if $\max =96 \%$, we can get:

$$
S(i)=(0.96 \cdot \ln (x-i)) / \ln (x-0.96)
$$

Using equation (14), we can obtain the Level number $j=8$, 5,4 , quantitative and qualitative value relationship $(S(1) \neq 100 \%)$

TABLE IV: THE NUMBER OF LEVELS J=8

\begin{tabular}{ccccccccc}
\hline \multicolumn{10}{c}{ TABLE IV: THE NUMBER OF LEVELS J=8 } \\
\hline$S(i)(\%)$ & 1 & 2 & 3 & 4 & 5 & 6 & 7 & 8 \\
\hline
\end{tabular}

TABLE V: THE NUMBER OF LEVELS $J=5$

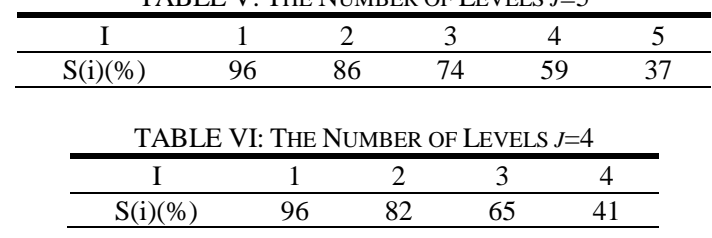

Obviously, if the maximum $S(i)$ is not $100 \%$, the number of levels is reduced to a minimum number. This maximum $S(i)$ is less than the given max. Based on the analysis above, the quantitative method is suitable for the highest quantitative value of $100 \%$, otherwise, the method only can be used in the number of levels high enough.

With the same method, after each level of the quantitative value obtained, the evaluation results of each grade of membership vector can be turned into a final score. The evaluation results are treated as the formation of the weight vector (normalization), and then weighted average with the evaluation results to obtain the final score. For example, the results of an evaluation class membership vector (assumed to be descending order $)$ is $B=(0.38,0.18,0.16,0.16,0.12)$, with a 1-100 points scale to grade quantitatively: $V=[100,90,77,61,39]^{T}$. Then a total score is: $S=B \cdot V=80.96$ (scores).

\section{CONCLUSION}

Because assessment model for students' learning ability results reflect the level of learning for the students and have important implications for individual development, people should pay more attention to get reliable evaluation. Thus, which would provide a reference for the teaching management. The next step is to explore the model of concrete practice application. Based on fuzzy comprehensive evaluation model, we can also evaluating students' intercultural competence. Though the existing comprehensive evaluation methods, such as the analytic hierarchy process (AHP), back propagation neural networks (BP-NN), gray system theory (GSE), data envelopment analysis (DEA), and fuzzy comprehensive evaluation (FCE), are commonly used in many different fields, the complex issues of multilevel indicators still need a better approach.

\section{REFERENCES}

[1] N. Raghavendra and R. Rajini, "Engineering education: Innovative practices and future trends (AICERA)," in Proc. 2012 IEEE International Conference, July, 2012. pp. 1-6.

[2] P. D. Umbach and M. R. Wawrzynski, "Faculty do matter: The role of college faculty in student learning and engagement," Research in Higher Education, vol. 2, pp. 153-156, Feb. 2005.

[3] L. A. Zadeh, "Fuzzy sets," Information and Control, vol. 8, no. 1, pp. 338-353, 1965.

[4] J. H. Chen and Z. Wei, "A model of multi-objective comprehensive evaluation for power plant projects," in Proc. the CSEE, vol. 22, no. 12, pp. 152-155, 2000.

[5] X. T. Gong and Y. G. Fu, "An evidential reasoning approach for college students' autonomous learning ability evaluation," in Proc. 5th International Symposium on Computational Intelligence and Design, pp. 410-414, 2012.

[6] Q. Feng, "The synthesis evaluation model on student's ability of using information technology in economic management profession in colleges," in Proc. (BMEI), 2011 International Conference on Business Management and Electronic Information, vol. 3, pp. 760-763, May 2011. 
[7] W. Tan, "The application of fuzzy level evaluation in occupational ability evaluation for higher vocational students," Computer Science and Electronic Technology International Society, vol. 5, 2013.

[8] J. P. Liu, Z. G. Wang, and R. H. Yi, "Scientific talent evaluation system based on combining ability and performance," in Proc Asia-Pacific Humanities and Social Sciences Conference(APHSS 2014), vol. 6 , 2014.

[9] D. Gurer, M. Desjardins, and M. Schlager, "Representing a student's learning states and transitions," in Proc. the 1995 American Association of Artificial Intelligence Spring Symposium on Representing Mental States and Mechanisms, Stanford, 1995.

[10] W. L. Wang, Artificial Intelligence and Its Application, Beijing: Higher Education Press, 2005.

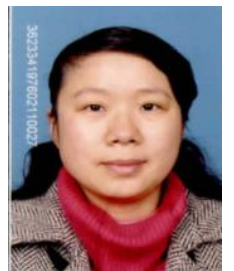

Yan Cheng was in February, 1976 in Wu Yuan City She got the bachelor's degree in 1998, the master's degree in 2005, and the Ph.D. degree in 2010 from Tongji University in Shanghai and she is now a postdoctor of Tongji University in 2015, majored in computer science, engaged in e-learning, virtual learning community and educational technology innovation. From July 2013 to March 2014, she went to the California University as a visiting scholar. Cheng's main research interests include intelligent computer aided education, educational data mining, virtual learning community and e-learning.

Currently she is a postdoctor of Tongji University and a professor in Jiangxi Normal University in Nanchang, Jiangxi province. As a researcher, currently she is the leader of 2 national NSFC (Natural Science Foundation of China) Plan Project and more than 5 provincial projects on IT and education innovations. She is the author of more than 30 scientific papers, won Guanghua Ph.D. scholarship, 3 talks in the global intelligent automation conference, 1 national doctoral academic best paper award. She published 1 academic monograph (Beijing, Science Press, 2014) on educational data mining, 5 computer professional teaching materials.

Dr. Cheng is the senior member of the Systems Engineering Association of China, the review expert of Computer Application Study, one member of Chinese Intelligent Automation, the consulting evaluation expert of small and medium-sized enterprise in Jiangxi province. Dr. Cheng is also the direction leader of the ministry of education "software engineering" discipline and the expert in education informationization.

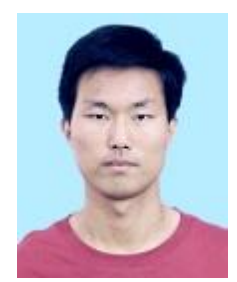

Zhi Ming Yang received the bachelor's degree in June 2013 from Hubei University of Arts And Science, Hubei. Currently he is a master of computer science and technology of Jiangxi Normal University. His research interests include personalized study, virtual learning community and non-cooperative game.

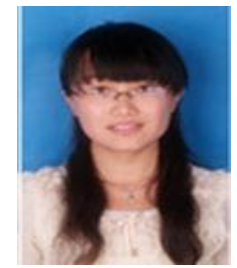

Yan Zeng was born in September 1990. She is studying the master of computer science and technology of Jiangxi Normal University. And her research direction is e-learning and behavior analysis. As the team members of tutor, she actively participates in a number of researches, interested in complex system and network learning behavior. 